This item was submitted to Loughborough's Research Repository by the author.

Items in Figshare are protected by copyright, with all rights reserved, unless otherwise indicated.

\title{
Qualification of academic facilities for small-scale automated manufacture of autologous cell-based products
}

PLEASE CITE THE PUBLISHED VERSION

http://dx.doi.org/10.2217/rme.14.47

PUBLISHER

(C) Future Medicine

VERSION

AM (Accepted Manuscript)

\section{PUBLISHER STATEMENT}

This work is made available according to the conditions of the Creative Commons Attribution-NonCommercialNoDerivatives 4.0 International (CC BY-NC-ND 4.0) licence. Full details of this licence are available at: https://creativecommons.org/licenses/by-nc-nd/4.0/

\section{LICENCE}

CC BY-NC-ND 4.0

\section{REPOSITORY RECORD}

Hourd, Paul C., Amit Chandra, David Alvey, Patrick J. Ginty, Mark J.S. McCall, Elizabeth Ratcliffe, Erin Rayment, and David J. Williams. 2017. "Qualification of Academic Facilities for Small-scale Automated Manufacture of Autologous Cell-based Products". figshare. https://hdl.handle.net/2134/25986. 


\title{
Qualification of academic facilities for small-scale automated manufacture of autologous cell-based products
}

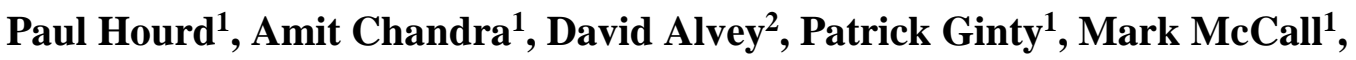 \\ Elizabeth Ratcliffe ${ }^{1}$, Erin Rayment ${ }^{1}$, David J. Williams ${ }^{1}$ \\ ${ }^{1}$ EPSRC Centre for Innovative Manufacturing in Regenerative Medicine, Centre for Biological Engineering, \\ Loughborough University, Leicestershire, LE11 3TU, UK. ${ }^{2}$ Kitenics Ltd, Northamptonshire, PE8 4AL, UK. \\ Corresponding Author: Professor David Williams, EPSRC Centre for Innovative Manufacturing in \\ Regenerative Medicine, Centre for Biological Engineering, Loughborough University, Leicestershire, LE11 \\ 3TU, UK. E-mail: D.J.Williams2@lboro.ac.uk
}

\begin{abstract}
Academic centres, hospitals and small companies, as typical development settings for UK regenerative medicine assets, are significant contributors to the development of autologous cell-based therapies. Often lacking the appropriate funding, quality assurance heritage or specialist regulatory expertise, qualifying aseptic cell processing facilities for Good Manufacturing Practice (GMP) compliance is a significant challenge. The qualification of a new Cell Therapy Manufacturing Facility (CTMF) with automated processing capability, the first of its kind in a UK academic setting, provides a unique demonstrator for the qualification of small-scale, automated facilities for GMP compliant manufacture of autologous cell-based products in these settings. This paper shares our experiences in qualifying the CTMF, focussing on our approach to streamlining the qualification effort, the challenges, project delays and inefficiencies we encountered and the subsequent lessons learned.
\end{abstract}

\section{Key Words:}

ATMP, autologous, automation, cell therapy, GMP, manufacturing, qualification, regulation, validation. 


\section{Introduction}

In Europe, the major stakeholders developing cell-based therapy products are academic centres, hospitals, charitable organisations and small companies. Academic and clinical centres with Good Manufacturing Practice (GMP) compliant facilities are now significant contributors to the development and manufacture of investigational autologous cell-based therapies [1-5]. In the UK, a small number of GMP manufacturing facilities have been established within major academic institutions and teaching hospitals. Many of these are licensed by the Medicines and Healthcare product Regulatory Agency (MHRA) to manufacture cellular therapies for use in clinical trials or to make small-scale batches of material for prescribing on a named patient basis under the UK 'Specials' licence [6,7]. Others are in the design, build or validation phase prior to regulatory accreditation.

Currently undergoing validation, the new purpose built Cell Therapy Manufacturing Facility (CTMF), housed within the Centre for Biological Engineering (CBE) at Loughborough University, UK is an example of one such facility. Funded by Loughborough University, with the support of the Engineering and Physical Sciences Research Council (EPSRC) and the former East Midlands Development Agency (emda), the design, construction and commissioning of the CTMF was completed in conjunction with a specialist cleanroom engineering company in February 2009. Designed for multi-patient autologous product manufacturing, the CTMF, with the 'GMP specified' CompacT Cellbase automated cell culture system (TAP Biosystems) at its core, provides cleanroom facilities to Grade B standard, with separate production areas and equipment for both manual and automated cell culture in Grade A environments [8] (Figure 1 \& 2).

Under the European directives regulating the manufacture of Advanced Therapy Medicinal Products (ATMPs) in which the principles of GMP apply [8,9], a programme of work to 
qualify the facility and the CompacT Select automated cell culture system began in March 2010. The objectives were to establish and provide documented evidence that the premises, equipment, utilities and operating systems were designed in accordance with the requirements of GMP (Design Qualification; DQ) and are built, installed and operate in compliance with their design specification (Installation Qualification (IQ)/Operational Qualification (OQ)).

At the time, the ATMP regulations were evolving and their interpretation also uncertain [10]. Only a small number of academic GMP qualified facilities were operational in the UK with none having automated cell processing capability. Changes in the regulatory landscape have continued, evidenced by recent revisions to the European guidelines for GMP and process validation [11-13]. Moreover, other potentially far reaching developments in the existing ATMP regulation, reported recently by the European Commission, now envision a more favourable environment for ATMP developers in academic settings and significantly, recognise the specific characteristics of autologous products [14,15]. Against this backdrop, the CTMF qualification project provides an opportune and unparalleled demonstrator for the qualification of small-scale facilities for the GMP compliant, automated production of autologous cell-based products in academic, hospital or small company settings.

This paper shares our experiences as we progressed along the facility qualification pathway. It focuses on our approach to streamlining the qualification effort, the challenges, project delays and inefficiencies we encountered and the subsequent lessons learned. The paper is formatted into four main phases of the qualification programme that breakdown into a number of lessons learned within each phase, highlighting those specifically related to qualification of the CompacT Cellbase.

\footnotetext{
< Insert Figure 1 here>

$<$ Insert Figure 2 here $>$
} 


\section{Project Initiation}

\section{Develop a clear business strategy and secure long-term Institutional buy in}

The availability of the skills and infrastructure for the CTMF project grew out of the industry supported academic research carried out at Loughborough University during the EPSRCfunded remedi project [16]. At the time, the regenerative medicine industry in the UK was still in its infancy as a commercial entity. Academic resources beyond the initial investment in the infrastructure were unpredictable and to an extent remain so. Establishing a clear business strategy and long term project plan from the outset was therefore unrealistic. In fact, academic uncertainties deferred the formation of a formal validation team until a year after the physical completion of the facility. The combined effect was to preclude any advantages to be gained from fully integrating the validation requirements into the facility design specification and engineering phases of the project at inception. Embodying the economic realities of academic and hospital settings this effectively defined the following progressive and integrated approaches to both facility qualification and business strategy development throughout the project.

\section{Structure and maintain continuity of the project team}

The critical first step in any qualification project is the formation of a project team that has the appropriate experience, knowledge and expertise in the relevant specialised fields such as automation, Quality Assurance (QA) and regulatory compliance. These skill sets are often rare in academic settings and may need to be drawn, at least in part, from consulting or engineering firms or by leveraging existing strengths in other academic or clinical centres $[5,17]$. We for example augmented the project team with external QA expertise, but otherwise were able to call on the relevant core competencies from in-house expertise in automated cell culture and regulated industry experience in GMP manufacturing [18]. 
These individuals will form the core team or decision making Validation Steering Committee (VSC) having intersecting roles at both the management and implementation levels. At the management level, the committee's remit is to oversee the qualification programme and coordinate the critical interfaces with the UK regulator (MHRA), the primary collaborators (i.e. TAP Biosystems) and institutional and funding stakeholders. At the implementation level, a small operational and technically qualified sub-team of the committee needs to be constituted and empowered with full decision making authority to plan, prioritise, coordinate and execute the facility and equipment qualification activities. Whatever the team organisation, it is important to establish individual roles, functions, interdependencies and levels of authority at the outset. In academic settings, this helps avoid conflicts with commitments that team members may have to other academic activities as the project progresses and also situations where personnel move to other research projects before the completion of the programme.

Regular meetings of the VSC ensure continued open communication, planning and coordination between team members throughout the duration of the project. Their scheduling and agenda is typically compelled by critical project milestones. Adding fixed business and regulatory update items to the core agenda makes sure that active business development regulatory/industry standards intelligence activities are captured. This assures that business, regulatory and operational requirements relevant to the phase of the qualification programme are aligned and best practice employed going forward. Likewise, the structure of these meetings is important. Our experience shows that a risk and science based approach to cohesive decision making and consequent action-led problem resolution is essential to ensuring timely and cost-effective project completion. Detailed minutes of each meeting provide an informal incremental change record that can be used to simplify reporting to 
stakeholders. Parenthetically, they also provide a robust validation project history on which much of this paper is based.

\section{Involve a Quality Assurance (QA) function from the start}

Augmenting the core VSC and sub-team with a QA function at the outset widens the knowledge, expertise and oversight in areas of GMP, regulatory compliance and industry trends. This ensures a clear understanding of the qualification scope and that quality practices and procedures are incorporated into the project from the start.

\section{Map the process of facility qualification}

Usually, the outcomes of a facility qualification project impact several key stakeholders and involve activities requiring input from multiple suppliers, contractors or service providers. Basic quality management tools such as the suppliers-inputs-process-outputs-customers (SIPOC) diagram can be usefully applied to build a high level mutual understanding of the project scope and workflow and agree its boundaries. In identifying the feedback and feedforward loops between the expectations of the customers (stakeholders), suppliers and the critical components of the qualification programme, it allows the project team to construct the logical sequence and staging of all the key activities and deliverables. For example, the execution of the environmental monitoring protocol by the validation team $(\mathrm{P})$, is triggered by the delivery of specified supplies of swabs, settle and contact plates (I) from the supplier (S). Samples of airborne and surface viable particles collected during execution of the process are packaged $(\mathrm{O})$ for transport to an external vendor for incubation and microbiological assessment (C). The sub-processes or activities that make up the environmental monitoring protocol specify and convert the inputs into the outputs.

If the principle of 'as simple as you can, complex as you must' is applied this approach can help maximise the efficiency of limited internal resource in terms of the level of effort in both 
performance and documentation of activities. Equally, it helps resolve external resource requirements, in terms of minimising the time consumed in securing materials, supplies and services necessary to perform activities. It can also assist the project team in predicting disruptions so that project risks are recognised early and mitigation efforts can be initiated in time.

\section{Project Planning: Plan the work, challenge it and plan it again}

\section{Adopt a modular validation strategy}

In academic settings, interventional funder timelines centred on capital expenditure can result in a time lag between the completion of the facility construction or upgrade and the formation of the validation team. This can prevent an orderly turnover of the facility to the validation team. Under these circumstances, a modular validation strategy, based for example on the ISPE’s Baseline ${ }^{\mathrm{TM}}$ Guide to Commissioning and Qualification [19], can be utilised to address these shortcomings. As part of the modular validation platform, building a primary document base, although not mandatory from a regulatory perspective, provides a means of defining the facility, equipment and utilities, together with the manufacturing processes capable of being carried out in the facility.

The qualification activity is derived from these supporting documents, the components of which are shown in the top panel of Figure 3. On one level, they provide the foundation and supporting evidence to confirm the physical completion of the facility, while also providing an entry point and reference to the facility design specification and for facilitating a structured design review of the facility. On another level, they allow the engineering specifications on which the IQ/OQ is usually based to be related to the process requirements (documented in production and process rationales/maps) and the risks critical to product quality and safety. Under the modular approach, these rationales drive the qualification effort 
and provide clarity on what does and does not need to be qualified. When fully integrated with a Quality Management System (QMS), a modular approach reduces the complexity associated with some traditional top heavy validation approaches. It eliminates unnecessary qualification effort and provides a more concise Master Validation Plan (MVP) that is easier to understand and implement.

\section{<Insert Figure 3 here $>$}

\section{Complete a regulatory requirements analysis}

A formal comprehensive review and analysis of the European regulatory framework for the manufacture of ATMPs under GMP is essential [8-10]. It confirms aspects of the facility, equipment, utilities and practices involved in the manufacturing process that are critical to product quality and safety. Identifying gaps or areas where clarification of the regulatory intent and compliance expectation is required, particularly when dealing with novel automated systems or settings, provides the impetus and scope for early dialogue with the MHRA. In defining the qualification scope and methodology, the analysis allows the project team to begin to build the facility MVP and identify the components of the QMS required for GMP compliance (Table 1).

\section{Implement a document management system from the start}

Establishing an electronic, controlled access document management system is an integral part of the early planning process and a three-phase construction of the QMS. Phase one focusses on the control system requirements. The target of this phase is to establish the primary validation policy and procedures, training and change control documentation and to implement the supporting document management system. This provides an early workable system for change management. Likewise it lays the foundation for determining critical timings for the preparation and delivery of the documentation or data required for the 
operational (Phase two) and management system (Phase three) requirements and for qualification activities to progress.

\section{Establish early consultation with the MHRA and an interface with Institutional stakeholders}

Known to be a critical issue in academic and hospital settings, strengthening the interface between the site/building operational management systems and the facility with respect to security, maintenance and control of critical HVAC (heating, ventilation and air conditioning) systems and their air handling units (AHUs) is a priority. This implies that building a reliable relationship with the Institutional Facility Management function is vital. Our experience shows that this can require a protracted phase of cross-learning at multiple organisational and operational levels. It needs to be an integral part of the planning process from inception of the project. An early judgement is needed on the level of the site planned preventative maintenance (PPM) required and the feasibility of its delivery under existing institutional facility management arrangements.

A recent European survey of academic facilities involved in the development and production of ATMPs has indicated that regulatory advice is essential for successful conversion to GMP and clinical trial [5]. Similarly, with a novel automated processing system on the one hand and regulatory uncertainty on the other, early consultations that the CTMF validation team (with the TAP Biosystems design team) had with the MHRA in the project initiation phase were critical. By confirming GMP compliance expectations and identifying specific areas expected to be subjected to regulatory scrutiny, this helped define the scope of the facility qualification effort and was crucial to understanding how automation fits with the GMP production of autologous cell-based therapies (Table 1).

Designed to automate the maintenance and expansion of cells in T-flasks from up to 90 individual patients in parallel, specific questions were raised about the potential for cross- 
contamination within the CompacT Cellbase processing area; a critical area for all autologous

processing. Addressing these concerns presented significant scientific and practical

challenges. The solution was to develop a novel challenge test that could be applied

specifically to a risk based evaluation of potential cross-contamination pathways within a

controlled environment GMP setting i.e. that does not in itself create the potential for

contamination of the environment in which it is used [see Box 1 for experimental details].

The utility of this challenge test turned out to be a decisive component of the CompacT

Cellbase design qualification, obviating the need for major design changes.

\section{Box 1: CompacT Cellbase Challenge Testing: A novel simulation test system for evaluating cross-contamination within GMP controlled processing environments}

Supported by a detailed Failure Modes Effects Analysis (FMEA) of the system, challenge experiments were designed to evaluate CompacT Cellbase processing areas and risks associated with potential for cross-contamination, aerosol creation and micro-droplet formation. We developed a novel challenge test using Glo Germ ${ }^{\mathrm{TM}}$ as a means of detecting cross-contamination, applicable to testing an automated cell culture platform within a GMP regulated environment. Glo Germ ${ }^{\mathrm{TM}}$ is a commercially available hygiene product used for teaching aseptic techniques (www.glogerm.com). It comprises $5 \mu \mathrm{m}$ melamine copolymer resin beads that fluoresce under Ultra Violet light illumination and has been used previously in novel cytometry applications to measure aerosol containment in high velocity cell sorters when sorting potentially biohazardous samples [20,21]. Advantages of using Glo Germ $^{\mathrm{TM}}$ are that it is non-biohazardous, inexpensive, requires no specialist microbiological knowledge or handling of bacteria, has minimal preparation time $(<30 \mathrm{~min})$ and ancillary reagent requirements, requires no incubation and provides potentially quantitative and immediately available results [20].

All standard system components used in CompacT Cellbase cell processing (pipetting, pouring, shaking, swirling, pooling, capping, decapping etc.) were challenged separately using a full system usage protocol. Glo Germ ${ }^{\mathrm{TM}}$ density was determined from a stock solution of Glo Germ ${ }^{\mathrm{TM}}$ (1g in $100 \mathrm{~mL}$ ethanol) by filtering triplicate samples $(25 \mathrm{~mL})$ of serially diluted Glo Germ ${ }^{\mathrm{TM}}$ solution $\left(10^{-8}\right.$ dilution, $0.22 \mu \mathrm{m}$ filter) and detecting the number of particles retained on the filter by microscopy. The detectable particle density from $1 \mathrm{~g}$ of Glo Germ ${ }^{\mathrm{TM}}$ powder in $100 \mathrm{~mL}$ ethanol was $8 \times 10^{9}$ particles ( $\mathrm{n}=3, \mu \pm \sigma=20.1 \pm 3.8$ particles $/ 25 \mathrm{~mL}$ diluted solution. Qualitative assessment was performed before and after each challenge run by draping a black-out cloth to cut-out light to the CompacT Cellbase processing cabinet and using a UV light the critical processing areas were visually inspected for the presence / absence of Glo Germ ${ }^{\mathrm{TM}}$ particles. Quantitative assessment was performed on all flasks by filtering contents through a $0.22 \mu \mathrm{m}$ filter and microscopic assessment of filters for counts of Glo Germ $^{\mathrm{TM}}$ particles. No visible signs of Glo Germ ${ }^{\mathrm{TM}}$ particles were detected inside the robot cabinet or on any of the filter discs. 


\section{Installation and Operational Qualification Readiness}

\section{Employ an enhanced design review}

In academic or hospital settings, facilities are often transitioning from non-GMP facilities or facilities accredited as tissue establishments. Limited capital and manpower resources can lead to delays in the turnover of new or renovated facilities to the validation team. These situations, especially where regulatory compliance expectations are in a state of flux, place greater emphasis on the design qualification and design review of the facility.

The start of this activity calls for a 'shakedown' phase to ensure the design and build of the facility reflects its intended use. Involving a documentation gap analysis and a physical walkthrough and inspection of the facility build by the validation team, this phase targets the identification of minor design errors or deficiencies and areas of non-compliance requiring remedial work or implementation of procedural controls. At the same time it confirms where design elements are still to be installed or commissioned in the facility. This permits detailed comparisons of the functional design and physical arrangement of the facility with the User Requirement Specification (URS), GMP and Health \& Safety requirements to be made in a formal Design Qualification (DQ) protocol (DQ, while not a regulatory requirement, refers to the documented verification that the design of the facilities, systems and equipment is suitable for the intended purpose). The DQ provides the platform for a rigorous structured design review by the VSC. Under a change control process, the resulting remedial plan enables the validation deliverables and mitigating actions required to address compliance and quality gaps to be prioritised prior to the execution of IQ (pre-IQ) or OQ (pre-OQ). This avoids further modifications to the facility or equipment later in the qualification phase which might otherwise evoke numerous deviations and change controls resulting in delays and increased expenditure. 


\section{Effect a system impact assessment}

Aligned with the ISPE guidance [19], a system impact assessment of the key design elements identified during the DQ phase can be used to streamline the qualification programme. If supported by technological risk assessments rather than just generic criteria, critical aspects as they relate to product quality and safety, can be used to better classify equipment/systems as critical (and/or having direct impact) or non-critical (and/or indirect impact). Decisions relating to the extent of qualification then provide the opportunity to finalise the MVP and determine the level of resource and effort required to execute the plan.

\section{Establish facility operational status levels}

Imposing strict cleaning and facility access regimes do little to promote adherence to cleaning protocols and consistency of results. Rather, the facility maintenance effort and cost can be rationalised by removing non-value added practices during the qualification programme. In the CTMF for example, this was achieved by establishing four facility operational status levels at the start of the project (Figure 4). These operational levels were constituted to manage both the risk and the economies of scope for CTMF operations, primarily in terms of the degree and frequency of cleaning, the level of dress code/gowning demanded for facility entry and the rate of consumption of energy, materials and consumables e.g. $\mathrm{CO}_{2}$ gases for incubators, liquid nitrogen for cryostores etc.

\section{$<$ Insert Figure 4 here $>$}

\section{Establish a facility contamination control plan}

Central to GMP, the risk-based contamination control plan lays the foundation for maintaining the facility in a state of control as it transitions between operational levels during the qualification programme. Risk identification needs to follow a holistic approach, screening the whole facility environment for factors and risk nodes influencing the outcomes of automated aseptic processing and the levels of contamination (Figure 5). The target of this 
step is to identify all potential threats and relevant vulnerabilities within this environment so that an assessment of the contamination and cross-contamination risks can be made e.g. using risk management and assessment tools such as cause and effect diagrams, FMEA etc. The facility contamination control plan makes use of this data to identify the sources of contamination, routes of transfer and mechanisms of control or risk mitigation specific to each of the operational levels. The plan identifies the control mechanisms necessary to address the potential risks of contamination. Whether by facility design and qualification, procedural control or the enactment of cleaning/disinfection, dress code or training regimes, these risk control mechanisms need to become critical elements of the IQ/OQ protocols.

\section{Streamline the amount of paperwork}

The paperwork and administrative burden associated with GMP is a significant hurdle for small academic manufacturers [5]. Documentation practices that do not add value to how documents are created and controlled lead to misapplication of the project team's time to areas that have little impact on the ability of the system to meet GMP requirements. Several approaches can be adopted to streamline the amount of qualification paperwork. Our experience shows that in combination the following approaches serve to improve document preparation, review and approval turnaround times and can be effective in minimising deviations and time-consuming explanations of errors that can otherwise lengthen and make qualification efforts less convincing when audited. These include: the use of qualification protocols designed and structured to capture outstanding actions from previous qualification activities, which obviate the need for elaborate deviation systems; the establishment of document review systems with clear expectations of the value added by the reviewer (e.g. technical, regulatory, scientific or editorial) prior to submission; and the creation of functional specification documents to support OQ protocol development for each item of installed equipment. Furthermore an overarching review system is needed to ensure an 
ongoing evaluation of areas for improvement by capturing explicit knowledge and experience gained from document implementation and execution.

\section{Perform activities in the right sequence}

Once a compliant facility design has been finalised following the design review, a step-wise approach to the qualification phase is needed to maximise the efficiency of internal resources and resolve external resource requirements and logistics. This is necessary to verify site acceptance testing (SAT) readiness for selected systems, ensure efficient transition between IQ and OQ and minimise delays to execution. It can be achieved by a sequenced IQ of each room in the facility using structured room IQ protocols. As the final major system audit of the facility prior to OQ, this is the critical step by which the qualification, maintenance, calibration, training and supply chain activities can be prioritised, such that they and associated second level tasks are performed in the right sequence prior to OQ.

\section{Maximise facility control and operating efficiencies before OQ of critical or direct impact equipment}

The implementation of a facility environmental monitoring programme is necessary to heighten the level of oversight and control of the facility, utilities and critical equipment before starting the OQ. This should be guided by the relevant International Standards (e.g. ISO14644-1). Automated Facility Management Systems (FMS) offer advantages over manual systems in that they allow regular surveillance of continuous, real-time data from multiple located sensors. By measuring trends in non-viable particles and pressure differentials for example, this provides a routine diagnostic assessment of facility infrastructure (Figure 1). It gives the validation team early warnings of operational inefficiencies or contamination problems such that the relevant risk can be recognised early and mitigation actions initiated before OQ activities can be disrupted or compromised (Table 1). 
A parallel activity is to establish baseline airborne and surface viable particle levels under static conditions in the unmanned 'at rest' state. At this stage, rather than setting high targets for acceptable levels of 'viable contamination' or bioburden, it is better to adopt a pathway approach to establish 'reference points' before and after periods of major activity. This is sufficient to provide a measure of the state of facility control and allows the effectiveness of cleaning/disinfection regimes to be verified. Likewise it identifies opportunities to exclude redundant or unnecessary steps in the process of cleaning/disinfection and to scale down the bioburden sampling plan. The latter can significantly reduce costs, especially if microbiological testing needs to be outsourced.

<Insert Figure 5 here $>$

\section{Installation and Operational Qualification Execution}

\section{Integrate commissioning and qualification activities where possible}

Following good engineering practice [19], pre-validation work carried out by the engineering contractor or vendor, including factory acceptance testing (FAT), site acceptance testing (SAT) and commissioning, can be leveraged to support the IQ/OQ programme. The CompacT Cellbase system, for example was subjected to a separate commissioning and qualification (IQ/OQ) programme conducted by the vendor under the demands of their own Quality Systems, e.g. Good Automated Manufacturing Practice (GAMP). Under these circumstances, activities to integrate the vendor's process with the facility qualification effort under its own validation policy require special attention. This ensures that shared requirements are managed by both the facility validation team and the vendor. As detailed in Table 1, integration of the pre-validation work into the Compact Cellbase and facility qualification activity yielded some important lessons with regard to maximising the efficiency of limited internal resources and streamlining the qualification effort. 
Commissioning verifies that the equipment was correctly installed, properly connected with site or facility services (e.g. liquid nitrogen supplies) and can be operated as specified and intended. Our experience shows that formal commissioning following good engineering practice [19] provides opportunities to leverage vendor expertise for operator training and transfer of best practice. For complex critical or direct impact systems in particular, the opportunity to test systems and components in all modes of operation is advantageous. It ensures that an assessment of the impact of the individual components on each of the other system elements is included in OQ protocols (e.g. as integrated components of the liquid nitrogen (LN2) systems, protocols for qualifying a controlled rate freezer (CRF) and vapour phase LN2 cryostore need to include an assessment of the impact of using the CRF on the capability of the cryostore to maintain temperature). Moreover, a pre-run of the OQ testing can be exploited to simplify I/OQ protocols, minimise errors or deviations in their subsequent execution and ultimately streamline the OQ process and its documentation. In cases where installed equipment has had an extended period of equipment downtime, a pre-OQ inspection routine (i.e. document and component verification) is advocated. This is necessary to bring the equipment on-line and verify that protocol requirements can be met and tested or have not changed due to component substitution or to design changes unknown to the validation team. This avoids potential IQ/OQ failures or deviations.

Most of the temperature controlled equipment in the CTMF met their thermal OQ testing acceptance criteria under the relevant ISO International Standards (e.g. BS EN 60068-35:2002). However in some cases equipment either needed to be modified (e.g. door seals replaced on freezers), their controls needed to be adjusted (e.g. set points adjusted, controlling probes relocated or shelves relocated for better circulation) or procedures for use needed to be changed in order to meet certain acceptance criteria (e.g. resulting from inadequate performance characteristics in terms of temperature gradients or thermal recovery). 
These findings are likely to be all too common in facilities where the URS for temperature controlled equipment has not been properly verified for its intended use before being procured. The inappropriate installation of household fridges is a frequently encountered example. At best, this can lead to a situation where revalidation of the equipment is necessary or at worst acceptance of fridges and freezers having qualified working spaces and hence storage capacities well below expectation (Figure 6).

\section{$<$ Insert Figure 6 here $>$}

Apply risk-based protocol design to reduce risk in the execution of complex aseptic process simulation procedures

Typically aseptic process simulation or media fill studies are logistically complex and time consuming even when performed using less personnel-intensive aseptic processing in automated systems such as the CompacT Cellbase. Operational qualification for this type of functionally closed system is not simply a matter of testing multiple automated aseptic manoeuvres (e.g. dispense, aspirate, swirling, shaking, etc.). It requires a holistic understanding of the whole process flow and of how the process design approach interacts with the operator, facility, supporting equipment and operational practices. This includes the whole process of vaporised hydrogen peroxide (VHP) bio-decontamination, machine set up, reagent and controls preparation, machine loading, priming and calibrating, flask processing, machine unloading, flask examination and analysis.

For instance, working with the vendor our approach was to take a systems view of the process and facility interfaces to identify process vulnerabilities likely to emerge under the operational practices of the CTMF (Figure 5). This incorporated a risk and science based analysis of operational and logistical conflicts as well as the manual interventions likely to pose the greatest risk of product contamination during the media fill (reagent/control preparation, loading the CompacT for example). The outcome was a media fill protocol with 
a defined and logical sequence of process steps and a system of checkpoints to guide the operator and checker through the process while also providing a record of compliance (resembling a manufacturing batch record).

The process simulation study, involving three consecutive automated aseptic media fills (30 T-175 flasks/run) using a sterile microbiological growth promoting medium as a surrogate product, was completed successfully but not without significant issues. Equipment or utility malfunctions (e.g. the CompacT Cellbase, BSC, VHP equipment, HVAC systems); unpredictable supply chain issues, operational deficiencies or technical issues disrupted or caused multiple delays to the start of the programme (by over 2 months in total). Such events can result in costly remedial or revalidation work and/or delays to the schedule while supplies are replenished or key services provision rescheduled. Detailed further in Table 1, these experiences illustrate how aseptic media fill issues can emerge as a consequence of a breakdown in one or more elements of GMP and yield important lessons for protocol design and execution.

<Insert Table 1 here $>$

\section{Plan for the unexpected - 'if anything can go wrong, it will'}

Our experience shows that small scale academic facility qualification programmes may expect to encounter periodic disruptions or delays of several months due to operational failures or unexpected events. These may be attributed to a combination of supply chain issues or equipment malfunctions, but more likely to recurring failures, malfunctions or unscheduled shutdowns of the HVAC systems that will disrupt air flow and supply quality e.g. pre-filter failures/blockages, air intake blockages, supply fan failure, heating/cold water coil failure etc.

From the CTMF perspective for example, the most disruptive of these events resulted from an unforeseen occurrence in the winter of 2011 when extremely low overnight air 
temperatures exposed weaknesses in the building infrastructure. A cascade of events that led to flooding and structural damage to the CBE building housing the CTMF and severely compromised the HVAC systems, delayed the start of the facility qualification programme by 6 months while the physical space was recovered and environmental control re-established. This scenario illustrates the need to establish risk mitigation strategies and contingency plans as an integral part of business continuity planning and for defining facility financial liabilities and insurance premiums or claims.

\section{Actively manage the supply chain risk}

The development of a reliable supply chain should be a primary concern for small academic manufacturers. Technical and financial diligence of critical vendors is a part, but the scale of and risk to IQ/OQ activities will not usually warrant extending this oversight to the selection and qualification of materials/consumables suppliers until later stages of the validation plan. It is therefore important to establish relationships with critical component suppliers and service providers early in the project initiation phase.

Locating specialised services and accredited suppliers of appropriately packaged or treated and often custom fabricated materials or components suitable for GMP can be a significant challenge, especially if the supply base is immature. Typically, pivotal aseptic media fill programmes can be exposed to most of this supply chain risk, as exemplified by some of the issues we experienced (Table 1). In some cases goods may not be available at the volumes required or require costly supply contracts at inappropriate scales of volume. It is also common for there to be only one accessible source of the material/consumable or service. Together, this makes the development of sufficient short-term inventories or contingency supply sources of critical materials difficult. It can also present logistical challenges to establishing windows for off-site, particularly non-local service provision e.g. for microbiological testing, irradiation of tubing sets and consumables etc. 
Even with plans to reduce exposure to supply shortages and extended lead times associated with delivery of specialised materials or services, supply chain issues can still result in increased costs and delays. Experience shows that suppliers may fail to deliver goods or services on time through a combination of unpredictable service delivery waiting lists, inaccurate vendor-quoted lead times or the prioritisation practices of some suppliers and service providers towards low volume purchasers. Institutional processing of purchasing documents is another common cause for delay.

With inadequate storage space, infrequent production runs and periods of inactivity likely to be a feature of small academic GMP facilities, these experiences illustrate the challenges and threats to developing a sustainable supply chain and purchasing cycle. If such facilities are to avoid exposure to supply chain risk and control high transactional costs associated with outsourced services, mitigation strategies will need to be incorporated into supply chain development, especially during the later stages of the qualification campaign. Mutual value propositions and service level agreements, supported by more appropriate policies for Institutional purchasing and tendering of GMP supplies will be a critical part of this.

\section{Maximise control of the HVAC systems - confront the 'elephant in the cleanroom'}

Part of the reality of validating an academic or hospital GMP facility is the management of the risks conferred by a lack of adequate control over Institutional building management or operational systems and the consequent exposure to unpredictable critical system failures or malfunctions that compromise environmental control. Engaging an external engineering specialist under a secondary level service agreement is one way of reinforcing oversight of critical HVAC systems. Our experience shows however that despite improved security and maintenance of critical HVAC systems, IQ/OQ activities can continue to be disrupted or put at risk by intermittent disturbances in the air flow and supply quality, primarily due to local 
and site-wide operational management and HVAC control system deficiencies. Not only do these events interrupt pre-OQ cleaning schedules, with consequent delays to the start of OQ and cleaning validation activities, they also have knock on effects. For example, by increasing costs related to non-value added repeat cleaning programmes and the loss of supplies (cleaning materials/media and consumables) due to expiry or deterioration of packaging resulting from overextended storage in often uncontrolled environments.

Institutional facility management functions within academic or hospital settings often lack the flexibility under Institutional policies and practice; have a blurring of responsibilities at the institutional level and poor understanding of the commercial and academic risks involved. Knowledge and understanding of this situation is the basis for establishing realistic expectations for achieving the level of control needed to maintain reliable HVAC system performance and facility air cleanliness. If Institutional service provision is to be prevented from falling short of delivering the required level of control of the HVAC systems, early decisions need to be made that consider (1) the feasibility of either strengthening primary service level agreements under existing institutional facility management arrangements or (2) deploying external specialist resources for PPM and control of HVAC systems under existing or new independent building management systems.

\section{Next Steps}

An operational and strategic review of the CTMF is required to revalidate the business case and redefine the business model framework. The organisational requirements, potential process development services and/or product offering need to be determined. Likewise the costs of sustaining business continuity and a GMP compliant facility under the scope of the operational transition framework, either within or outside the current Institutional infrastructure needs to be evaluated. This review will also need to factor in the impact of 
recent revisions to the European guidelines for GMP [11-13] and other potential developments in the ATMP regulation $[14,15]$.

\section{Conclusions}

As a complex, administratively-intensive, costly and time consuming undertaking, qualifying cell processing facilities for GMP compliance in small-scale, resource limited academic, hospital or small company settings can be a significant challenge, especially if they have limited pre-existing QA or manufacturing infrastructure [5]. This demonstrator project describes the process and pitfalls involved to highlight what it takes to set-up and develop an infrastructure for implementation of a facility qualification programme and the problems and constraints that the validation project team has to manage. This emphasises the additional and significant challenges of qualifying a novel automated processing system. Based on our experience, several practices are described that demonstrate potential opportunities to rationalise project resources and reduce or eliminate costly delays and time consuming nonvalue adding qualification activities. The lessons learned and insights gained come together to stress the following headline propositions: (i) An effective risk based approach to product quality, patient safety and regulatory compliance throughout the qualification planning is required to ensure that critical areas are addressed and resources are not consumed by nonvalue adding qualification activities; (ii) Incremental development of the facility must be accepted. This requires regular review, an infrastructure for continuous improvement and critically efficient change control mechanisms to be established as early in the project as possible; (iii) If an effective GMP facility is to be established, particularly in novel settings and immature markets, the validation team needs to extend their knowledge, understanding and control of supplies (both goods and services), information and financial flows across the entire upstream supply chain; (iv) For novel systems and settings, early and continued consultation with the regulator is a key component to identifying prevailing and future GMP 
and regulatory compliance expectations and defining the scope of the qualification effort; (v)

An understanding of the failure modes of critical or direct impact systems and utilities is required to specify effective testing, risk control and maintenance strategies that can improve their reliability and availability for qualification operations. As a postscript, although this occurs at an increased capital cost, if we had implemented these 'good maintenance practices' at the start of project we may have avoided many of the logistical delays we experienced due to system or equipment downtime associated with their diagnosis, trouble shooting, and repair.

Addressing the array of challenges and constraints described in this paper was critical to successful facility and CompacT Cellbase qualification. It is hoped that the experiences and practices described will serve as a guide for other academic, hospital or small companies as they plan new or upgraded facilities for GMP compliant automated or manual manufacture of autologous cell-based therapies.

\section{Executive summary}

\section{Project Initiation}

- Unpredictable resources in non-commercial settings define the need for progressive and integrated approaches to both facility qualification and business strategy development.

- Establish a project team with experience, knowledge and expertise in specialised fields of automated cell culture, quality assurance, GMP manufacturing and regulatory compliance

- Apply quality management tools to build a high level mutual understanding of the project scope and workflow, agree its boundaries and the expectations of customers and suppliers.

\section{Project Planning}

- Implement a modular validation approach to reduce project complexity and establish priorities.

- Complete a formal regulatory and GMP requirements analysis to identify gaps or areas and where clarification of the regulatory compliance expectation is required.

- Establish early consultation with the MHRA and an interface with the Institutional facility management function

\section{Installation and Operational Qualification Readiness}

- Employ a rigorous structured design review of the facility and a remedial plan to address compliance and quality gaps prior to the execution of IQ and OQ activities.

- Implement a risk-based contamination control strategy to maximise facility environmental control and operating efficiencies before OQ of critical or direct impact equipment. 
- Perform a sequenced IQ of each room in the facility so that equipment maintenance/calibration activities, implementation of risk controls (e.g. cleaning/disinfection, dress code and training regimes) and supply chain activities can be prioritised.

\section{Installation and Operational Qualification Execution}

- Use good engineering practice to leverage pre-validation work and vendor expertise to support the IQ/OQ programme.

- Develop risk-based challenge tests early in the project to evaluate the potential risks of contamination and cross-contamination in the automated autologous product processing area.

- Design and develop risk-based protocols for the execution of complex aseptic process simulation procedures based on learning from factory acceptance testing and trial runs.

- Actively manage vulnerabilities and exposure to upstream supply chain risks.

- Maximise control of the HVAC systems and their AHU units under existing institutional facility management arrangements or under new independent building management systems.

\section{Future perspective}

Building on the science and risk based International Conference on Harmonisation (ICH) guidelines, specifically ICH Q8, Q9, Q10 and Q11, new concepts and approaches to validation and change control are being promoted through U.S FDA and EMA initiatives and the work of ISPE and others [11,22]. New European process validation guidelines, adaptation of Annex 2 to ATMPs and impending revisions to Annex 15 of the European guidelines for GMP [11-13] put forward more progressive and graded validation pathways that will afford manufacturers new possibilities for manufacturing efficiency and flexibility.

Merging science-based risk management with an integrated Quality Systems approach will move validation away from traditional system-based approaches [19], placing greater reliance on process knowledge, understanding and control to assure product safety and efficacy. Experience from the pharmaceutical/biopharmaceutical industry suggests that the adoption of these approaches by the cell therapy industry will rely on advances in the state of current technology. Step changes in real time process monitoring capability and development of stable surrogate or reference cell lines and other industry standards, as a way of addressing Quality by Design like manufacturing design practice and continuous process verification approaches will be an integral part of this. Likewise advances in closed or functionally closed 
automated processing technology for cell manipulation, whether in the factory or hospital setting, will be a critical step. By reducing operator variation and eliminating the external environment, these systems will likely make validation of facility infrastructure and the manufacturing process intrinsically more straightforward and cost effective, besides being more amenable to change control and the demonstration of comparability [23].

The potential progress in some of these areas has been recognised in a recent report published by the European Commission in April this year following public consultation on the application of the Regulation (EC) No 1394/2007 on ATMPs [14,15]. Significantly, this report raises new possibilities for academic and other ATMP developers, not least with the recognition that the specific characteristics of autologous products and their manufacturing processes deserve specific consideration.

If the regulatory framework continues to adapt to rapid scientific progress and technological advances in cell-based therapy manufacturing, as an industry we will be able to derive and implement better and more effective ways of qualifying our facilities, equipment and automated manufacturing systems. Coupled with potentially far reaching changes to the ATMP regulation [15], this will ensure that our facilities and manufacturing systems are delivered fit for purpose and capable of supporting the reproducible manufacture of quality cell-based autologous therapy products across multiple manufacturing sites while controlling risk to patient safety.

\section{Acknowledgements}

The authors would like to acknowledge TAP Biosystems for providing technical and services support for the Compact Cellbase system.

Financial \& competing interests disclosure

Funding and support of the EPSRC is acknowledged. Initial funding was secured from its Innovative Manufacturing and the Life Sciences Interface Programmes both for the remedi 
Grand Challenge and its successor; the EPSRC Centre for Innovative Manufacturing in Regenerative Medicine. The construction of the CTMF was funded by Loughborough University and the former East Midlands Development Agency (emda). Support for the validation of the CTMF was provided by the Technology Strategy Board (TSB) and the Knowledge Transfer Account (KTA) of Loughborough University, funded by EPSRC.

The authors have no other relevant affiliations or financial involvement with any organization or entity with a financial interest in or financial conflict with the subject matter or materials discussed in the manuscript apart from those disclosed. No writing assistance was utilized in the production of this manuscript.

\section{References}

1. McKernon R, McNeish J, Smith, D. Pharma’s Developing Interest in Stem Cells. Cell Stem Cell. 6(6), 517-520 (2010).

2. Maciulaitis R, D’Apote L, Buchanan A, Pioppo L, Schneider CK. Clinical Development of Advanced Therapy Medicinal Products in Europe: Evidence that Regulators must be proactive. Molecular Therapy. 20, 479-482 (2012).

3. Culme-Seymour EJ, Davie LN, Brindley DA, Edwards-Parton S, Mason C. A decade of cell therapy clinical trials (2000-2010). Regenerative Medicine. 7(4), 455-62 (2012).

4. The Cell Therapy Catapult UK Clinical Trials Database as of November 2012 (2012). https://www.catapult.org.uk/documents/10726/1553967/CTC+database+commentary/0f818af38683-4b08-9815-d7ebdd092d4e (Accessed December 2013).

5. Pearce KF, Hildebrandt M, Greinix H, et al. Regulation of advanced therapy medicinal products in Europe and the role of academia. Cytotherapy. 16(3), 289-297 (2014).

***Research paper describes the results of a European survey of academic facilities involved in the development and production of ATMPs to assess to impact of Regulation (EC) No. 1394/2007.

6. Sethe S, Batkai C, Dickinson A, Hildebrand M. Manufacture of advanced therapies: Academia meets industry. Regulatory Rapporteur. 8(7/8), 9-10 (2011).

7. The ATMP Manufacturing Community Capacity Database: GMP Facilities licensed for ATMP manufacture in the UK as of December 2012 (2012). http://www.atmpmanufacture.org/Capacity/Capacity.php (Accessed December 2013).

8. Eudralex - Volume 4 Good Manufacturing Practice (GMP) guidelines for medicinal products for human and veterinary use. European Commission. http://ec.europa.eu/health/documents/eudralex/vol-4/ (Accessed April 2010).

9. Commission Directive 2003/94/EC of 8 October 2003 laying down the principles and guidelines of good manufacturing practice in respect of medicinal products for human use and investigational medicinal products for human use (2003). http://ec.europa.eu/health/files/eudralex/vol-1/dir_2003_94/dir_2003_94_en.pdf (Accessed April 2010).

10. Regulation (EC) No 1394/2007 of the European Parliament and of the Council of 13 November 2007 on advanced therapy medicinal products and amending Directive 2001/83/EC and Regulation (EC) No 726/2004 (2007). http://eurlex.europa.eu/LexUriServ/LexUriServ.do?uri=OJ:L:2007:324:0121:0137:en:PDF (Accessed April 2010).

11. Guideline on process validation for finished products - information and data to be provided in regulatory submissions. European Medicines Agency (2014). 
http://www.ema.europa.eu/docs/en_GB/document_library/Scientific_guideline/2014/02/WC5001 62136.pdf (Accessed April 2014).

12. EudraLex - Volume 4 Good Manufacturing Practice (GMP) guidelines for medicinal products for human and veterinary use. Annex 2. Manufacture of biological active substances and medicinal products for human use (2013). http://ec.europa.eu/health/files/eudralex/vol-4/vol4-an2__201206 en.pdf (Accessed April 2014).

*This revised guideline provides guidance for the interpretation of the principles and guidelines of GMP (GMP) for medicinal products. It contains specific adaptations for ATMPs.

13. EudraLex - Volume 4 Good Manufacturing Practice (GMP) guidelines for medicinal products for human and veterinary use. Annex 15. Qualification and Validation (2014).

http://ec.europa.eu/health/files/gmp/2014-02_pc_draft_gmp_annex.pdf (Accessed April 2014).

* This draft Annex is expected to be adopted in October 2014. It describes the principles of qualification and validation which are applicable to the facilities, equipment, utilities and processes used for the manufacture of medicinal products.

14. Regulation (EC) No. 1394/2007 on Advanced Therapy Medicinal Products. Summary of the response to the public consultation. European Commission (2013).

http://ec.europa.eu/health/files/advtherapies/2013 05 _pc atmp/2013 $04 \quad 03$ pc summary.pdf (Accessed November 2013).

15. Report from the Commission to the European Parliament and the Council. European Commission (2014). http://ec.europa.eu/health/files/advtherapies/2014_atmp/atmp_en.pdf (Accessed April 2014).

**Report published by the European Commission that takes stock of the situation of ATMPs in the EU and analyses the impact of the Regulation on advanced therapies. The report takes account of the public consultation on the application of Regulation (EC) No. 1394/2007.

16. Remedi: building a viable regenerative medicine industry - a guide for stakeholders. Williams DJ, Archer R, Dent A (Ed.). Loughborough University, Loughborough, UK (2010). http://www.epsrc.ac.uk/SiteCollectionDocuments/Publications/reports/Remedi.pdf (Accessed February 2013).

17. Trounson A, DeWitt ND, Fiegal EG. The alpha stem cell clinic: A model for evaluating and delivering stem cell-based therapies. Stem Cells Translational Medicine. 1, 9-14 (2012).

18. Williams DJ, Thomas RJ, Hourd PC, et al. Precision manufacturing for clinical-quality regenerative medicines. Phil. Trans. R. Soc. A. 370, 3924-3949 (2012).

19. Pharmaceutical Engineering Guides for New and Renovated Facilities (Volume 5) Commissioning and Qualification. International Society of Pharmaceutical Engineering (2001).

20. Oberyszyn A, Robertson F. Novel rapid method for visualisation of extent and location of aerosol contamination during high-speed sorting of potentially biohazardous samples. Cytometry. 43, 217-222 (2001).

21. Perfetto S, Ambrozak D, Koup R, Roederer M. Measuring containment of viable infectious cell sorting in high-velocity cell sorters. Cytometry. 52A: 122-130 (2003).

22. Guidance for Industry. Process Validation: General Principles and Practices U.S FDA (2011). http://www.fda.gov/downloads/Drugs/Guidances/UCM070336.pdf (Accessed April 2012).

23. Hourd PC, Ginty P, Chandra A, Williams DJ. Manufacturing models permitting roll out/scale out of clinically-led autologous cell therapies: Regulatory and scientific challenges for comparability. Cytotherapy. Accepted for publication (2014).

**Research paper that proposes prospective manufacturing models for the scale out of clinicallyled autologous cell therapy products and tests their prospects with a selected panel of US and UK thought leaders. The paper identifies multi-site enablers for reproducible manufacture. 


\section{Figure Legends}

\section{Figure 1. Layout of the primary elements of the Cell Therapy Manufacturing Facility.}

Occupying a total space of about $70 \mathrm{~m}^{2}$, the facility comprises a manual operations room (equipped with fridge, freezer, incubator and a Biological Safety Cabinet (BSC)) and a machine room for automated aseptic manufacture (equipped with the Compact Cellbase and a fridge). Both rooms are classified as Grade B (shaded green), as defined by Annex 1 of the EU guidelines for GMP [8], and meet the physical containment level 2 requirements. The BSC and CompacT Cellbase provide Grade A classified environments (shaded red). The facility also includes a Grade C (shaded yellow) cryopreservation room (equipped with a controlled rate freezer and a vapour phase liquid nitrogen (LN2) cryostorage unit supplied via insulated vacuum lines from two 240L LN2 supply tanks located outside the controlled area); unclassified (shaded white) material storage areas and a pass through room (equipped with a freezer and a controlled temperature storage area $\left(4^{\circ} \mathrm{C}\right)$ ). In addition there are off-facility areas for writing-up, bulk storage of supplies and documents. Rooms are isolated from each other by sealed walls with no connecting doors. The facility has controlled access for entrance and enforces unidirectional traffic flow patterns for personnel, materials, products, and waste via transfer hatches and two change/gowning rooms (Grade B and Grade C). The HVAC systems and their AHUs are located in the plant room above the facility. The facility environment is continuously monitored via a local Facility Management System, including non-viable particle counts ( 0.5 and 5.0 $\mu \mathrm{m}$ ) in grade B rooms and in Grade A environments, pressure differentials between rooms within the facility, humidity and temperature in the automated processing room, as well as temperature and $\mathrm{CO}_{2}$ of storage units.

\section{Figure 2. Photograph of the Compact Cellbase system (TAP Biosystems).}

Located in the Grade B automated cell culture laboratory within the Cell Therapy Manufacturing Facility, the Compact CellBase is an automated system designed and tested to support the development and manufacture of autologous cell therapy products. The CompacT CellBase automates the maintenance and expansion of cells in T-175 flasks from multiple patients within a Grade A classified environment [8] behind a restricted access barrier system under negative pressure, protecting the operator and ensuring aseptic processing of the products. Design elements include; 10 pumps for programmable media and reagent dispense, automated temperature and $\mathrm{CO}_{2}$ controlled incubator for 90 T-175 flasks and automated cell counting and viability measurement.

\section{Figure 3. Schematic of the modular validation strategy.}

Shows primary document development for the separate related qualification programmes under independent validation plans (VP) defined in the Master Validation Plan (MVP). Grey highlights indicate the scope of facility qualification activities addressed in this paper. Glossary: $D Q=$ design qualification; IQ = installation qualification; $\mathrm{OQ}=$ operational qualification; $\mathrm{PQ}=$ performance qualification; $\mathrm{CV}$ = cleaning validation; $\mathrm{CSV}=$ computer system validation; $\mathrm{ASV}=$ analytical system validation; $\mathrm{AMV}=$ analytical method validation .

\section{Figure 4: Cell Therapy Manufacturing Facility Operational Status Levels.}

Key: Decommissioned level (out of service); Level 4 - Under validation (some controls will be in place whilst the facility is validated but no product will be present and therefore the risk is minimal); Level 3 - Hibernation (validated facility, no product or active materials present, minimal entry and exit); Level 2 - Operational (validated facility for storage and handling of product (in secondary 
packaging) and active materials); Level 1 - Aseptic manufacture (validated facility for cell culture operations, product processing, product handling in primary and secondary packaging).

Figure 5. System diagram of Cell Therapy Manufacturing Facility interfaces. Factors and risk nodes influencing outcomes of automated aseptic processing and levels of contamination.

Figure 6. Schematic of temperature sensor positions for Operational Qualification of Freezer. Thermal profile showing mean temperature $\left({ }^{\circ} \mathrm{C}\right)$ at each probe position and for the remote Facility Management System (FMS) probe $\left(-25.7^{\circ} \mathrm{C}\right)$. In general the temperature gradient shows a significant increase in the top half of the freezer particularly at the front compared to the bottom half, with mean temperatures outside the specification $\left(-20^{\circ} \mathrm{C}\right.$ to $\left.-30^{\circ} \mathrm{C}\right)$. Sensors positioned according BS (EN 600683-5:2002). Corner sensors secured approximately $50 \mathrm{~mm}$ in from chamber walls. 
Figures

Figure 1.

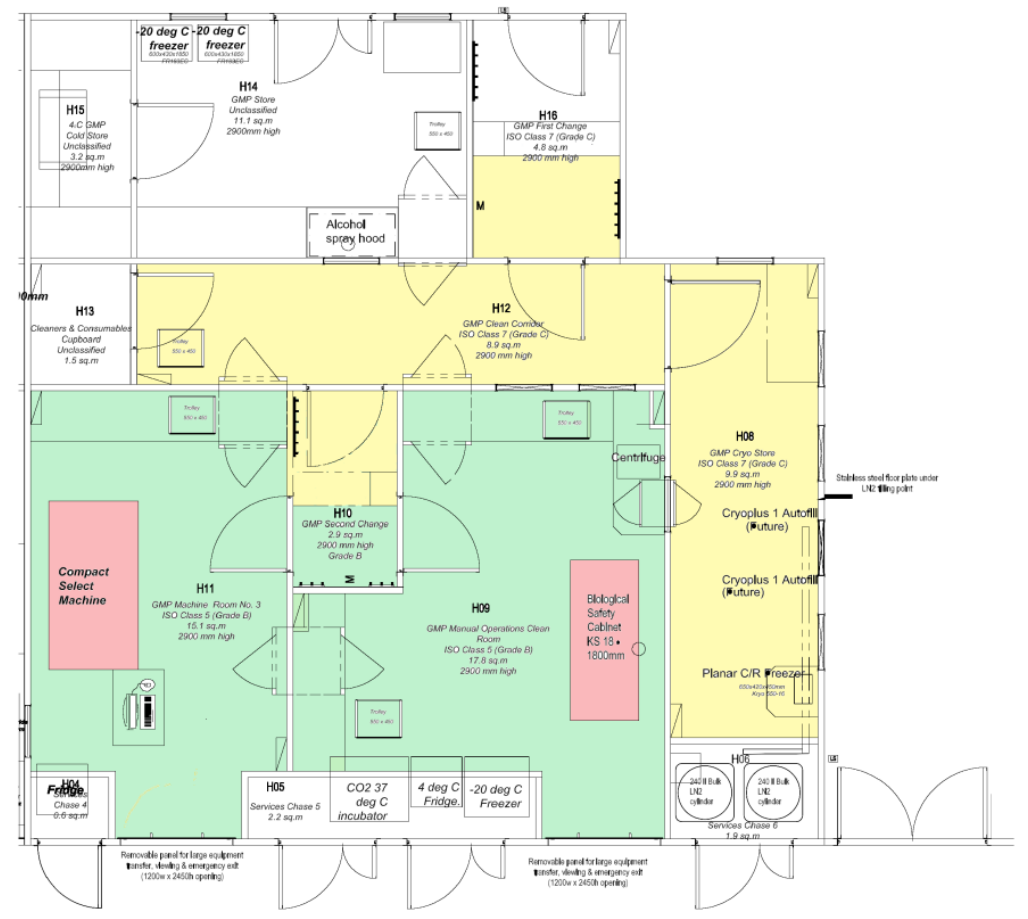

Figure 2.

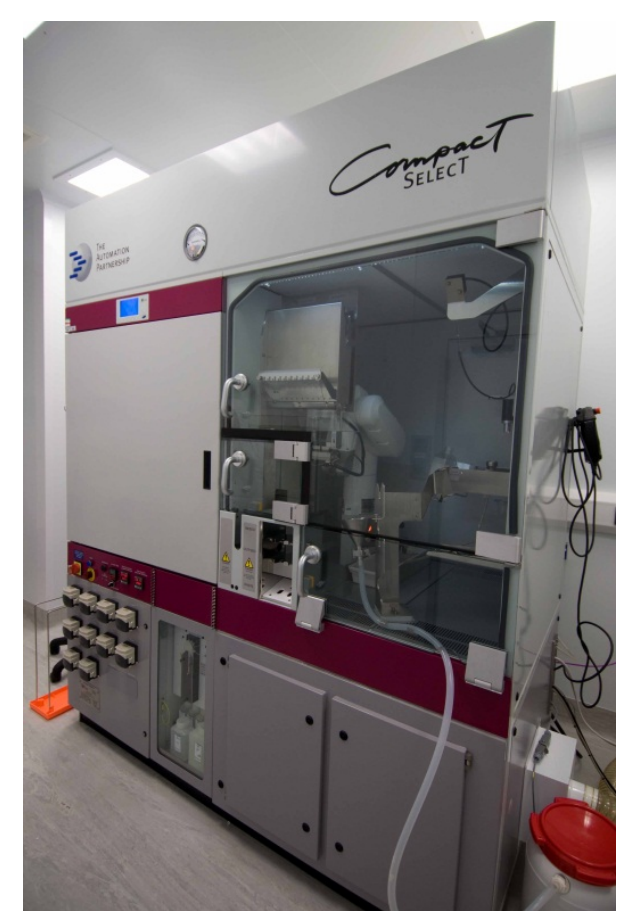


Figure 3.

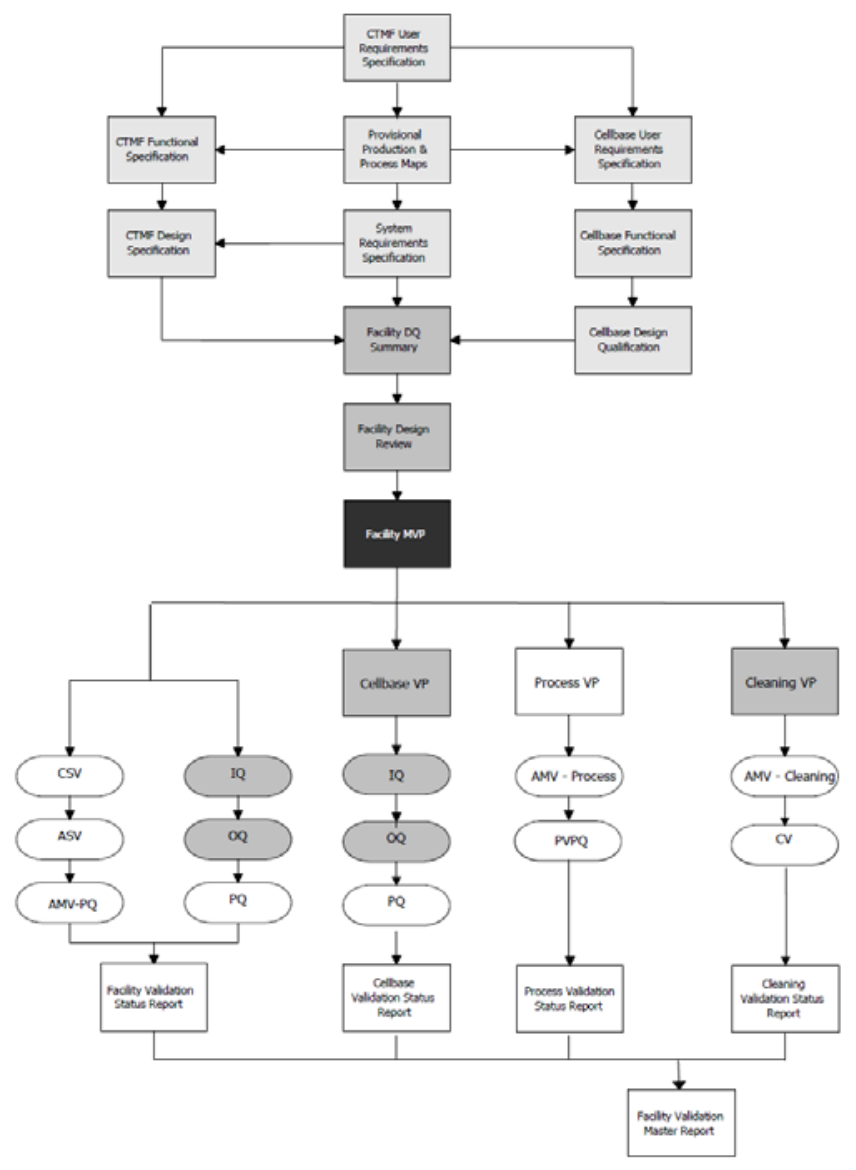

Figure 4.

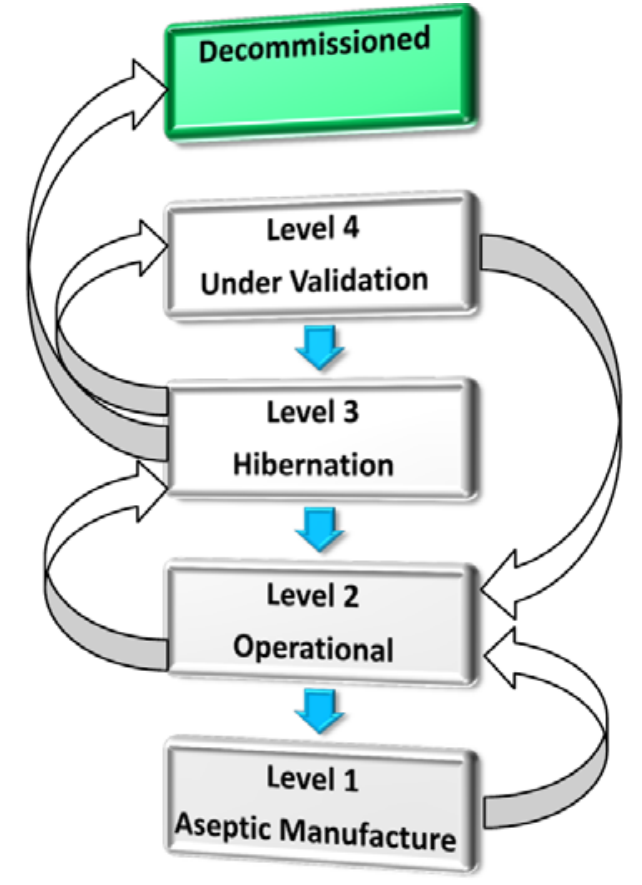




\section{Figure 5.}

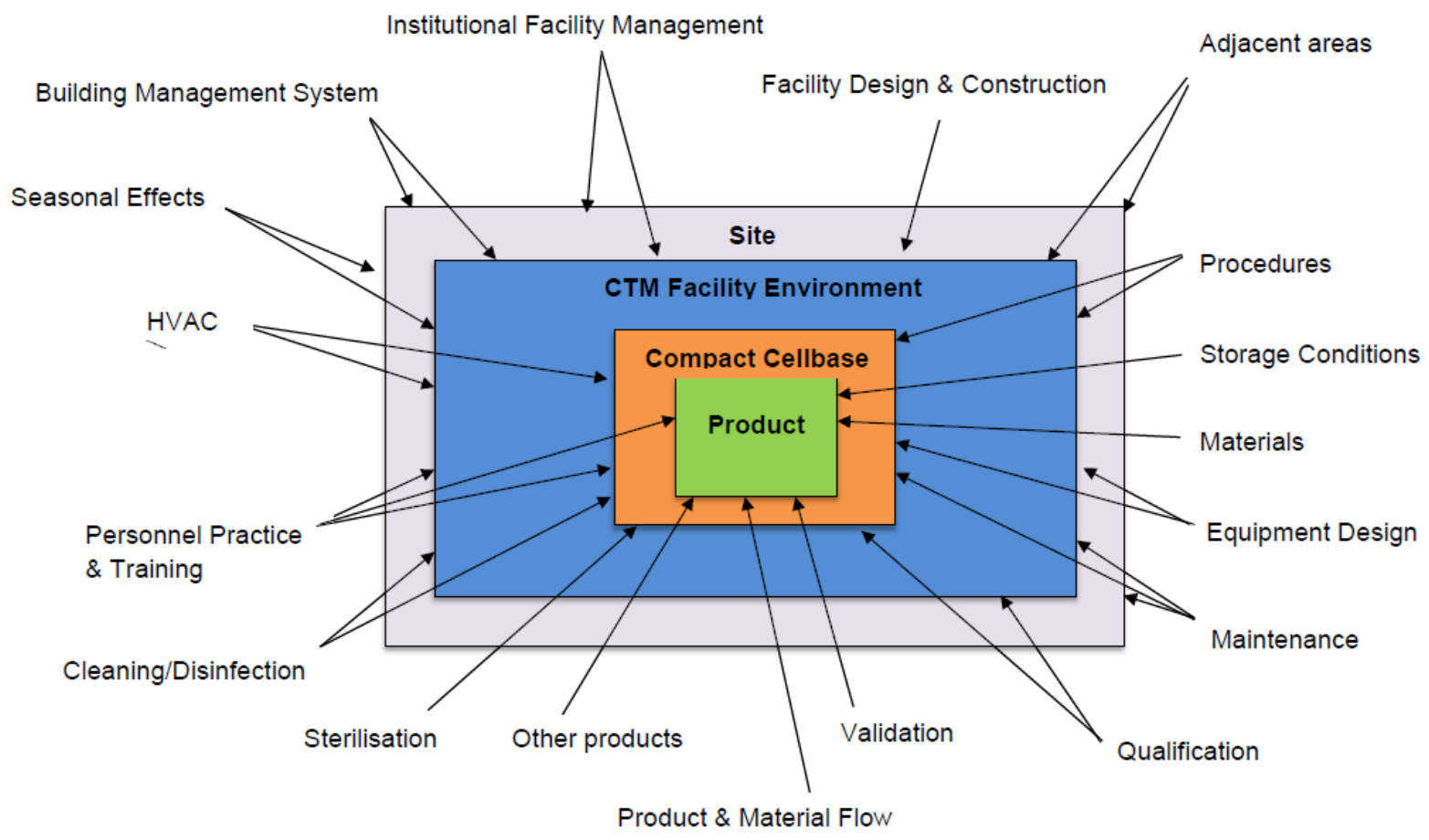

\section{Figure 6}

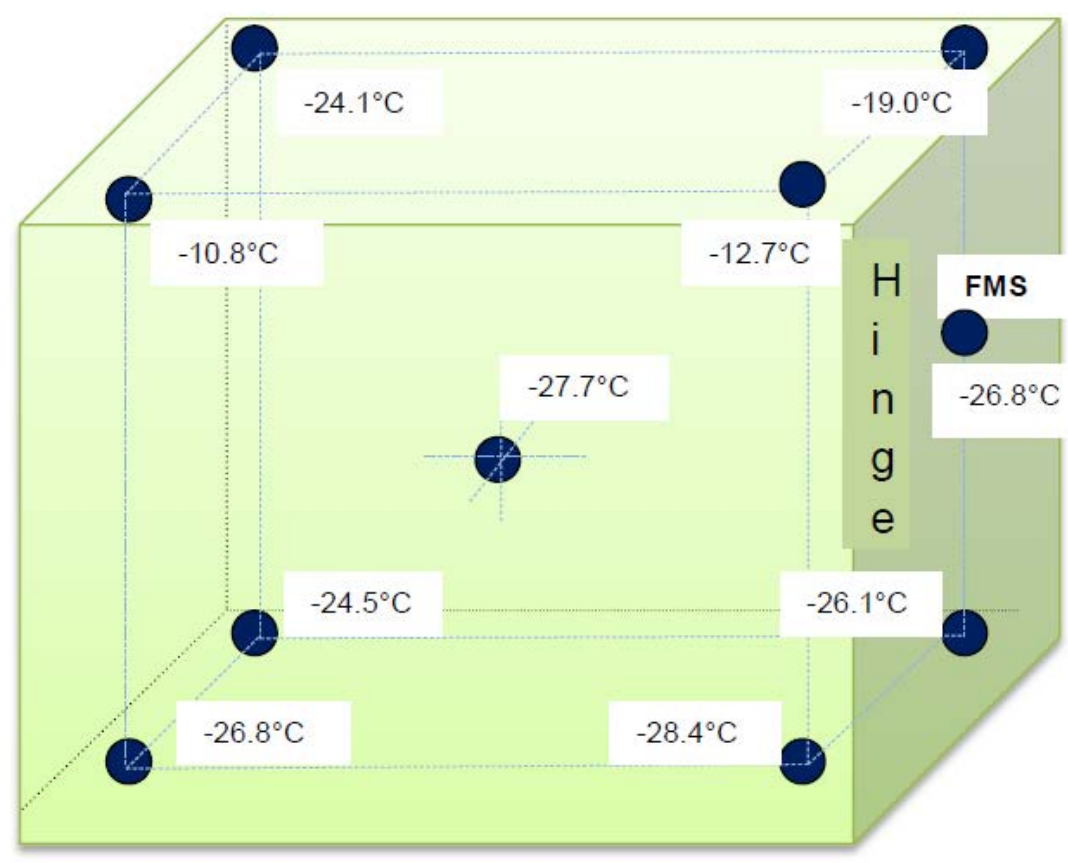


Table 1. Qualification of the CompacT Cellbase system: Major lessons learned from the perspective of the CTMF validation team

\begin{tabular}{|c|c|}
\hline Parameter & Summary of Lessons Learned \\
\hline $\begin{array}{l}\text { Regulatory } \\
\text { Requirements: } \\
\text { Analysis }\end{array}$ & $\begin{array}{l}\text { Conducting a regulatory requirement analysis early in the project initiation } \\
\text { phase was critical for identifying areas or gaps where clarification on how the } \\
\text { existing regulations apply to the qualification of the CompacT Cellbase system. }\end{array}$ \\
\hline $\begin{array}{l}\text { Consultation with } \\
\text { the UK Regulator } \\
\text { (MHRA) }\end{array}$ & $\begin{array}{l}\text { Early consultation with the MHRA was crucial to identifying critical CompacT } \\
\text { Cellbase system design features likely to come under the highest regulatory } \\
\text { scrutiny i.e. its air handling systems, its 'cleanability' and its integration with } \\
\text { the facility cleanroom and connected utilities. } \\
\text { As a critical component of the system Design Qualification (DQ), complex and } \\
\text { extensive risk-based challenge tests (Box 1) need to be developed to evaluate } \\
\text { the potential risks of contamination and cross-contamination in the automated } \\
\text { processing area. }\end{array}$ \\
\hline $\begin{array}{l}\text { Commissioning } \\
\text { and Qualification } \\
\text { Integration }\end{array}$ & $\begin{array}{l}\text { Following good engineering practice [19], coordinating and integrating the } \\
\text { factory acceptance testing (FAT), pre-delivery inspection (PDI), site acceptance } \\
\text { testing (SAT) and commissioning of the system with the DQ and design review } \\
\text { of the facility can realise cost and time savings in the qualification programme. } \\
\text { 'Hands on' involvement of members of the CTMF validation team in the } \\
\text { inspection and testing of the system during FAT and PDI at the vendors' site } \\
\text { was critical. Input from those with deep domain knowledge and experience in } \\
\text { manual aseptic culture processes and from a representative from the MHRA } \\
\text { was equally critical. } \\
\text { Involving this team in the pre-validation work allowed a quicker and more } \\
\text { efficient resolution of minor issues with the equipment and of compliance gaps } \\
\text { in the functional and physical arrangement of the facility. Delays that would } \\
\text { have resulted from discovering problems with the equipment or its installation } \\
\text { after on-site delivery were avoided. By identifying critical operational criteria } \\
\text { that require testing during the FAT/SAT the duration of the OQ was } \\
\text { significantly shortened. }\end{array}$ \\
\hline $\begin{array}{l}\text { Operational } \\
\text { Qualification (OQ) } \\
\text { Readiness }\end{array}$ & $\begin{array}{l}\text { Maximising facility environmental control and process critical equipment / } \\
\text { system operating efficiencies before the OQ of the CompacT Cellbase was } \\
\text { critical for successful execution. This included: } \\
>\quad \text { Implementation of risk control mechanisms (cleaning/disinfection, dress } \\
\text { code or training regimes) to address the potential risk of contamination as } \\
\text { part of a facility contamination control plan and the OQ protocol. } \\
\text { Prioritisation of the sequence of critical facility, equipment and utilities } \\
\text { SAT and OQ execution. } \\
>\quad \text { Surveillance of continuous environmental and facility infrastructure data to } \\
\text { provide early warnings of facility/equipment operational inefficiencies or } \\
\text { environmental disturbances (i.e. that may result in increased particle } \\
\text { counts). This allows early recognition of the relevant risk and mitigation } \\
\text { actions to be initiated before the OQ activity can be disrupted or } \\
\text { compromised. }\end{array}$ \\
\hline
\end{tabular}


Table 1. Continued...

\begin{tabular}{|c|c|}
\hline Parameter & Summary of Lessons Learned \\
\hline $\begin{array}{l}\text { Aseptic Process } \\
\text { Simulation }\end{array}$ & $\begin{array}{l}\text { Aseptic connections and manual manipulations during the set-up of the machine } \\
\text { by the operator are vulnerable to operator error and integrity failures in the } \\
\text { connector-tube line assemblies (e.g. media bag to machine). This can result in } \\
\text { leakages and potential exposure of media to contamination. Novel protocol } \\
\text { design approaches therefore need to involve a risk based evaluation of how } \\
\text { dynamic operational, ergonomic and human factors can interact. It is of note } \\
\text { that these interactions are often neglected by many suppliers/vendors. } \\
\text { The set-up and preparation of the CompacT Cellbase involves multiple manual } \\
\text { interventions. These include the aseptic loading, location, placement, } \\
\text { attachment and removal of equipment components and labware, such as waste } \\
\text { trays, tubing, indicator strips, pipette canisters, media bags, flasks and reagents } \\
\text { etc., Just as all these elements need to be tested during the FAT, extended } \\
\text { reliability tests should also be a key component in testing the multiple } \\
\text { automated manoeuvres involved. } \\
\text { Likewise, early learning or trial periods provide an opportunity to uncover and } \\
\text { correct unforeseen critical risk factors and process vulnerabilities, operational } \\
\text { deficiencies and logistical conflicts. } \\
\text { Operator training and proficiency testing of aseptic technique and measurement } \\
\text { capabilities (e.g. visual detection of turbid units) is advocated to reduce novelty } \\
\text { in the procedure, help drive error rates down and reduce the chance that } \\
\text { deviation will occur. }\end{array}$ \\
\hline $\begin{array}{l}\text { Supply Chain } \\
\text { Management }\end{array}$ & $\begin{array}{l}\text { As a logistically complex and extended (28 days) task, aseptic process } \\
\text { qualification is operationally difficult to schedule and is vulnerable to upstream } \\
\text { supply risks. } \\
\text { In immature markets, small-scale facilities using novel automated systems } \\
\text { should not underestimate the lead times and costs involved in procuring } \\
\text { specialised services (e.g. for VHP decontamination, irradiation of consumables, } \\
\text { bioburden testing) and locating accredited suppliers of appropriately packaged } \\
\text { or treated and custom fabricated materials or components suitable for GMP } \\
\text { (e.g. pre-filled culture media bags). } \\
\text { As a small scale academic facility, it may not be possible to establish mutual } \\
\text { value propositions or primary service level agreements with vendors. It is } \\
\text { therefore critical to establish informal relationships with suppliers of critical } \\
\text { goods or services early in the project, bearing in mind that the provision of } \\
\text { certain customised supplies e.g. appropriately packaged 'triple bagged' } \\
\text { consumable components such as flasks and pipettes, may require the supplier to } \\
\text { change their manufacturing processes and/or issue new products codes. } \\
\text { Likewise practices for institutional purchasing and tendering practices of these } \\
\text { specialist goods and services need to be developed if further delays are to be } \\
\text { avoided. }\end{array}$ \\
\hline
\end{tabular}

